\title{
Teoría de semigrupos fuertemente continuos de operadores lineales continuos aplicada a una ecuación de transporte-Difusión
}

\section{Theory of strongly continuous semigroups of continuous linear operators applied to a transport-diffusion equation}

\author{
LEANDRO JESÚS GALO ${ }^{1}$
}

Recibido: 14 de agosto de 2018/ Aceptado: 17 de noviembre de 2018

${ }^{1}$ Escuela de Matemáticas y Ciencias de la
Computación, Universidad Nacional Autónoma
de Honduras email: leandro.galo@ unah.edu.hn
In this work we apply the theory of semigroups of continuous linear operators to prove the existence and uniqueness of the solution for a certain transport-diffusion equation, in particular those that are strongly continuous, since they are generated by a linear operator usually denoted by $A$ and commonly called the infinitesimal generator of the semigroup. This operator is of great importance since it is usually the operator related to an equation or system of differential equations, that is, we relate the transport-diffusion equation with a linear differential operator that generates a strongly continuous semigroup, and thus apply certain known results in the area of the semigroups and that will allow us to prove the existence and uniqueness of the solution for our equation, in contrast with the classic methods known.

En este trabajo aplicamos la teoría de semigrupos de operadores lineales continuos para probar la existencia y unicidad de la solución para cierta ecuación de transporte-difusión, en concreto aquellos que son fuertemente continuos, ya que estos son generados por un operados lineal usualmente denotado por $A$ y llamado comúnmente el generador infinitesimal del semigrupo. Este operador es de gran importancia puesto que suele ser el operador relacionado a una ecuación o sistema de ecuaciones diferenciales, es decir, relacionamos la ecuación de transporte-difusión a un operador diferencial lineal, el cual genera a un semigrupo fuertemente continuo, y así aplicamos ciertos resultados conocidos en el área de los semigrupos y lo que nos permitirá probar la existencia y unicidad de la solución para nuestra ecuación, en contraste con los métodos clásicos conocidos.

PALABRAS CLAVES

Semigrupos, Operadores lineales, Ecuaciones diferenciales

KEYWORDS

Semigroups, Linear Operators, Differential Equations.

PACS

02.30.Jr 


\section{I | INTRODUCCIÓN}

T As ecuaciones diferenciales son de gran importancia en el modelamiento de muchos fenómenos de Lla ciencia y la ingeniería (ver (Evans, 2010; John, 1991)). La solución de tales ecuaciones puede ser útil en la predicción del comportamiento a futuro de un fenómeno, así como entender la teoría y funcionamiento de cierto proceso o la interacción entre los procesos subyacentes, la evaluación de ciertas hipótesis, la estimación de algún parámetro de interés, simulación computacional, e incluso hasta el control de dicho fenómeno. Por ejemplo, un modelo de crecimiento de un tumor puede ayudar a evaluar diferentes tratamientos, minimizando de esta forma el uso de ensayos clínicos sobre humanos o animales.

Uno de los mayores retos para los ingenieros y científicos al momento de usar ecuaciones diferenciales es cuando se necesita encontrar su solución. De hecho, en la mayoría de las aplicaciones prácticas resulta casi imposible encontrar una solución en forma cerrada (solución analítica). En tales casos resulta más factible el uso de métodos numéricos eficientes que aproximen la solución utilizando un computador digital (ver (Johnson, 2009, R. J. L. Lions, 2000a)).

Por otro lado, los matemáticos, además de trabajar en la construcción de modelos más reales y confiables, o en la búsqueda de nuevos y mejores métodos numéricos para resolver un problema de forma más apropiada, también trabajan en responder preguntas de interés teórico, pero de grandes implicaciones prácticas. Por ejemplo, ¿cómo se sabe si una ecuación diferencial tiene solución?. Muchos métodos numéricos pueden llevarnos a soluciones que en realidad no existen. Además, uno no quisiera gastar tiempo y esfuerzo al aplicar un método numérico sin saber si el problema tiene solución o no. Por otro lado, si se sabe que un problema tiene solución, ¿cómo saber si es única?. Típicamente, uno busca modelos cuya solución sea única. Para ilustrar esto, piense que Júpiter no puede tener dos trayectorias diferentes al mismo tiempo. Por otro lado, ¿cómo se sabe que una pequeña variación en los datos del modelo producirá una pequeña variación en la solución?. Esta pregunta es de gran importancia debido a que en la práctica uno nunca tiene los valores exactos de los datos (debido a los errores de medición y de redondeo). Uno quisiera saber si estas pequeñas variaciones producirán pequeñas diferencias en la solución (ver (Brezis, 2007, Evans, 2010; John, 1991)).

La existencia y unicidad de la solución de una ecuación diferencial puede probarse de diferentes formas, eso dependerá de las propiedades de la ecuación (si es lineal o no lineal, por ejemplo). El principio del máximo es una de las estrategias básicas para probar la unicidad. La existencia suele ser más complicada de probar (ver (Evans, 2010, John, 1991)). Este artículo se enfoca en estudiar la existencia y unicidad de la solución de una ecuación diferencial utilizando la teoría de semigrupos de operadores lineales fuertemente continuos.

En 1930 la teoría de semigrupos de operadores se comenzó a aplicar a ciertas ecuaciones diferenciales que están relacionada con un operador diferencial lineal, el cuál genera un semigrupo de operadores especiales llamado semigrupo de operadores fuertemente continuos. De esto último es de donde surgen nuevas técnicas que permiten la prueba de existencia y unicidad de soluciones para muchos problemas que en el sentido clásico eran difíciles de tratar. Teoremas propios de esta teoría como ser el de LumerPhillips y Hille-Yosida (ver (Curtain, 1978, R. J. L. Lions, 2000b; Pazy, 1983) ) son muy importante para dichas pruebas y resultan muy prácticos, en contraste con los métodos clásicos ya que se han aplicado a varias ecuaciones diferenciales, como ser la ecuación de calor, la ecuación de la onda, etc (ver (Ervedoza y Zuazua, 2010, R. J. L. Lions, 2000b, Pazy, 1983)). 
A su vez está teoría ha colaborado con el desarrollo de la teoría de control y las ecuaciones diferenciales como se puede ver en (J. L. Lions, 1988; Russell, 1978). Al hablar de problemas de control nos referimos de una manera muy general a lo siguiente:

Dado un fenómeno modelado por una ecuación o un sistema de ecuaciones diferenciales que comienza en un estado inicial, queremos determinar si existe un tipo de control que nos permita llevar al sistema a un estado final (el estado deseable) de una manera no forzada, asumiendo que existe solución única para el modelo. Además, nos preguntamos que tipo de costo implica controlar al sistema de la manera deseada.

La idea anterior es la base de la teoría de control y junto con la teoría de semigrupos ha permitido estudiar una gran cantidad de ellos, como por ejemplo el costo de control de problemas del tipo parabólico como se puede ver (Fattorini y Russell. 1971). Dicho problema sirvió de motivación para estudiar el costo de controlabilidad a cero para la ecuación de transporte-difusión que se presenta en este escrito (ver (Boyer, 2013; Galo, 2018; Guerrero, 2005)). Cabe destacar que la ecuación de transporte-difusión tiene gran importancia en áreas como termodinámica, fenómenos de transporte, entre otras (ver (Bird, Stewart, y Lightfoot, 2007; Michael Reed, 1978)).

El trabajo que presentamos a continuación está organizado de la siguiente manera. En la sección 2 presentamos algunos conceptos preliminares que sirven de base a la teoría de semigrupos. En la sección 3 definimos los semigrupos de operadores fuertemente continuos, así como algunas propiedades importantes que se aplican a las ecuaciones diferenciales. En la sección 4 presentamos las ecuaciones de transporte y transporte-difusión así como la prueba de la existencia y unicidad de las soluciones para cada una de ellas utilizando la teoría de semigrupos de operadores fuertemente continuos. Por último en la sección 5 realizamos nuestras conclusiones más relevantes.

\section{I PRELIMINARES}

Dado un operador lineal continuo $A \in \mathcal{L}\left(\mathbb{R}^{n}, \mathbb{R}^{n}\right)$ planteamos el siguiente sistema para $t \geq 0$, con $x_{0} \in \mathbb{R}^{n}$ un vector inicial dado

$$
\begin{gathered}
x^{\prime}(t)=A x(t), \quad x(t) \in \mathbb{R}^{n}, \\
x(0)=x_{0} .
\end{gathered}
$$

Es conocido que al ser $A$ un operador continuo la solución del problema anterior es de la forma $x(t)=\mathrm{e}^{t A} x_{0}$, donde $e^{A t}=\sum_{k=0}^{\infty} \frac{(t A)^{k}}{k !}$ es convergente para $t \geq 0$.

Es sabido que un operador lineal continuo es acotado, es decir, existe constante real fija $M>0$ tal que

$$
\|A x\| \leq M\|x\|
$$

Ahora ¿qué pasa si $A$ es un operador lineal no acotado definido en un subespacio de un espacio de Hilbert $H$ (ya que el caso acotado en $H$ es similar cuando consideramos el espacio $\mathcal{M}_{n \times n}(\mathbb{R})$ ) ¿será que la solución es parecida a la dada para el caso acotado? estudiamos a los operadores lineales densamente definidos, cerrados y disipativos, ya que estos generan semigrupos de operadores fuertemente continuos, los cuales son la herramienta idónea para cuando tenemos operadores no acotados asociados a un sistema de ecuaciones diferenciales. 
Sea $H$ un espacio de Hilbert complejo con producto interior $\langle\cdot, \cdot\rangle$ y sea $A: H \rightarrow H$ un operador lineal con dominio $D(A) \subseteq H$, tal que $A$ es densamente definido, es decir, $D(A)$ es denso en $H$.

Definition El operador lineal $A: H \rightarrow H$ es cerrado si el conjunto $\{(x, A x): x \in D(A)\}$ es un subconjunto cerrado en $H \times H$.

Definition El operador lineal $A$ es disipativo si

$$
\operatorname{Re}\langle A x, x\rangle \leq 0, \text { para todo } x \in D(A) .
$$

Definition $\mathrm{El}$ adjunto $A^{*}$ del operador lineal $A$ es un operador lineal dado por

$$
\begin{aligned}
A^{*}: \quad D\left(A^{*}\right) \subseteq H & \rightarrow H \\
y & \mapsto A^{*} y
\end{aligned}
$$

donde $D\left(A^{*}\right)$ es el conjunto de todos los $y \in H$ tales que el mapeo

$$
\begin{array}{cl}
D(A) \subseteq H & \rightarrow \mathbb{C} \\
x & \mapsto\langle A x, y\rangle
\end{array}
$$

es continuo; es decir, existe una constante $C>0$ dependiente de $y$ que satisface $|\langle A x, y\rangle| \leq C\|x\|$ para todo $x \in$ $D(A)$. Para cada $y \in D\left(A^{*}\right), A^{*} y$ es el único elemento de $H$ que cumple $\langle A x, y\rangle=\left\langle x, A^{*} y\right\rangle$ para todo $x \in$ $D(A)$.

Como observación al pedir que $A$ tenga dominio $D(A)$ denso en $H$, podemos aproximar a cualquier punto $x \in H$ por medio de una sucesión de elementos de $D(A)$ y esto nos permite trabajar sólo con elementos de $D(A)$ para después extendernos a todo $H$ por medio de sucesiones.

\section{I SEMIGRUPOS DE OPERADORES}

Definition Un conjunto $S$ no vacío con una operación binaria cerrada $*$ es llamado un semigrupo, si la operación binaria es asociativa, es decir, para $x, y, z \in S$ se cumple que $x *(y * z)=(x * y) * z$.

Un ejemplo sencillo sería el conjunto de matrices $\mathcal{M}_{n \times n}(\mathbb{R})$ con la operación usual de multiplicación de matrices.

Definition Una familia uni-paramétrica $S=\{S(t): H \rightarrow H \mid t \in[0, \infty)\}$ de operadores lineales continuos es un semigrupo de operadores continuos sobre $H$ si

I) $S(0)=\mathrm{Id}$, donde $I d: H \rightarrow H$ es el operador identidad en $H$.

II) $S\left(t_{1}+t_{2}\right)=S\left(t_{1}\right) \circ S\left(t_{2}\right), t_{1}, t_{2} \in(0, \infty)$.

III) Además decimos que $S$ es un $\mathcal{C}_{0}$-semigrupo o un semigrupo de operadores lineales fuertemente continuos sobre $H$ si

$$
\lim _{t \rightarrow 0^{+}} S(t) x=x \text { para todo } x \in H .
$$

Denotamos por $\mathcal{L}(H)$ al espacio de operadores lineales continuos en $H$ dotado con la norma de operador $\|\cdot\|_{\mathcal{L}(H)}$.

A continuación enunciamos los siguientes resultados, los cuales se pueden encontrar en (Curtain 1978, R. J. L. Lions, 2000b, Pazy, 1983). 
Theorem 1. Sea $\{S(t): H \rightarrow H \mid t \in[0, \infty)\}$ una familia de operadores lineales fuertemente continua, entonces existe $C>0$ y $\lambda \in \mathbb{R}$ tal que

$$
\|S(t)\|_{\mathcal{L}(H)} \leq C e^{\lambda t} \text { para todo } t \in[0, \infty) .
$$

Theorem 2. Sea $S=\{S(t): H \rightarrow H \mid t \in[0, \infty)\}$ un $\mathcal{C}_{0}$-semigrupo. Entonces el generador infinitesimal de $S$ es un operador lineal $A: D(A) \subseteq H \rightarrow H$ densamente definido y cerrado, tal que

$$
D(A):=\left\{x \in H: \lim _{t \rightarrow 0^{+}} \frac{S(t) x-x}{t} \text { existe }\right\} \text { y } A x:=\lim _{t \rightarrow 0^{+}} \frac{S(t) x-x}{t}, x \in D(A) .
$$

Si ponemos $x(t)=S(t) x_{0}$ con $x_{0} \in H$, entonces $x(t) \in C([0, \infty) ; H)$; más aún, si $x_{0} \in D(A)$ entonces $x \in C([0, \infty) ; D(A)) \bigcap C^{1}([0, \infty) ; H)$ y satisface

$$
\begin{aligned}
x^{\prime}(t) & =A(x(t)), \quad t \in[0, \infty), \\
x(0) & =x_{0} .
\end{aligned}
$$

Además el conjunto de operadores adjuntos $\left\{S(t)^{*} \mid t \in[0, \infty)\right\}$ de $S$ es un $C_{0}$-semigrupo con generador infinitesimal $A^{*}$, donde $A^{*}$ es el adjunto de A.

Notamos que el teorema anterior nos dice que si tenemos un $\mathcal{C}_{0}$-semigrupo y calculamos su operador infinitesimal, entonces el semigrupo proporciona la solución del sistema (1). En la práctica, por lo general nos dan el sistema asociado con el operador $A$, entonces nos preguntamos si existe un $C_{0}$ semigrupo cuyo generador infinitesimal coincida con A. En este sentido el teorema de Hille - Yosida nos dice cuando un operador lineal $A$ es el generador infinitesimal de un $\mathcal{C}_{0}$-semigrupo. Nos enfocaremos en el resultado de Lumer-Phillips para operadores densamente definidos, cerrados y disipativos que mencionamos a continuación.

Theorem 3. Sea A un operador densamente definido y cerrado, tal que tanto A como $A^{*}$ son disipativos. Entonces $A$ es el generador infinitesimal de un $\mathcal{C}_{0}$-semigrupo y para cada $x_{0} \in D(A)$, existe una única (solución) $x \in C^{1}([0, \infty) ; H) \cap C^{0}([0, \infty) ; D(A))$ tal que

$$
\begin{aligned}
& x^{\prime}(t)=A x(t), \quad t \in[0, \infty), \\
& x(0)=x_{0}
\end{aligned}
$$

además $\|x(t)\| \leq\left\|x_{0}\right\|$ para todo $t \in[0, \infty) y\left\|\frac{d x}{d t}(t)\right\|=\|A x(t)\| \leq\left\|A x_{0}\right\|$ para todo $t \in[0, \infty)$.

Enunciamos un resultado que resuelve el problema de Cauchy no homogéneo (ver (Curtain, 1978)).

Theorem 4. Sea A un operador lineal densamente definido y cerrado. Si A y $A^{*}$ son disipativos, entonces para cada $x_{0} \in D(A), T \in[0, \infty), y f \in C^{1}([0, T] ; H)$, existe una única (solución) $x \in C^{1}([0, T) ; H) \cap C^{0}([0, T) ; D(A))$ del sistema

$$
\begin{gathered}
x^{\prime}(t)=A x(t)+f(t), \quad t \in[0, T], \\
x(0)=x_{0}
\end{gathered}
$$

dada por

$$
x(t)=S(t) x_{0}+\int_{0}^{t} S(t-\tau) f(\tau) d \tau, \text { para todo } t \in[0, T] .
$$

Prosigamos a considerar al espacio de Hilbert $U$ dotado con el producto interior $\langle\cdot, \cdot\rangle_{U}$, lo llamaremos el espacio de controles, es decir, $u \in L^{2}((0, T) ; U)$ será una función de control para cierto problema de 
Cauchy definido más adelante.

Para el operador adjunto $A^{*}$ de $A$, dotamos a $D\left(A^{*}\right)$ con el producto interior

$$
\left\langle z_{1}, z_{2}\right\rangle_{D\left(A^{*}\right)}:=\left\langle z_{1}, z_{2}\right\rangle_{H}+\left\langle A^{*} z_{1}, A^{*} z_{2}\right\rangle_{H} \text {, con } z_{1}, z_{2} \in D\left(A^{*}\right) .
$$

Entonces $D\left(A^{*}\right)$ con este producto es espacio de Hilbert e induce la norma

$$
\|z\|_{D\left(A^{*}\right)}:=\|z\|_{H}+\left\|A^{*} z\right\|_{H} .
$$

Sea $D\left(A^{*}\right)^{\prime}$ el espacio dual normado de $D\left(A^{*}\right)$ con espacio pivote $H$, es decir, $D\left(A^{*}\right) \subset H \subset D\left(A^{*}\right)^{\prime}$. Consideramos el operador lineal $B: U \rightarrow D\left(A^{*}\right)$ que satisface las siguientes condiciones

(a) Existe una constante $C>0$, tal que

$$
|(B u) z| \leq C\|u\|_{U}\|z\|_{D\left(A^{*}\right)} \text { para todo } u \in U, z \in D\left(A^{*}\right) .
$$

(b) Para todo $T>0$ existe $C>0$ tal que se cumple la siguiente condición de regularidad

$$
\int_{0}^{T}\left\|B^{*} S(t)^{*} z\right\|_{U}^{2} \leq C_{T}\|z\|_{H} \text { para todo } z \in D\left(A^{*}\right)
$$

donde $B^{*}: D\left(A^{*}\right) \rightarrow U$ es el operador adjunto de $B$, es decir,

$$
\langle B u, g\rangle=\left\langle u, B^{*} g\right\rangle, g \in D\left(A^{*}\right), u \in U .
$$

(c) Dado que $S^{*}(t), t \in[0, \infty)$ es una familia de semigrupos fuertemente continua se puede probar que (3) es equivalente a la existencia de algún $T>0$ y una constante $C_{T}>0$ tal que

$$
\int_{0}^{T}\left\|B^{*} S(t)^{*} z\right\|_{U}^{2} \leq C_{T}\|z\|_{H} \text { para todo } z \in D\left(A^{*}\right)
$$

Definition Para $T>0, y_{0} \in H$, y $u \in L^{2}((0, T) ; U)$ consideramos el siguiente problema de Cauchy para los operadores $A$ y $B$,

$$
\begin{gathered}
\dot{y}=A y+B u(t), \quad t \in(0, T), \\
y(0)=y_{0} .
\end{gathered}
$$

Ahora presentamos la motivación para la definición de solución del sistema (6)-(7). Sea $\tau \in[0, T]$ y $\phi:[0, T] \rightarrow H$ una función diferenciable, si tomamos el productor interior en $H$ de 6 con $\phi$ e integramos sobre $[0, \tau]$ tenemos

$$
\langle y(\tau), \phi(\tau)\rangle_{H}-\left\langle y_{0}, \phi(0)\right\rangle_{H}-\int_{0}^{\tau}\left\langle y(t), \dot{\phi}(t)+A^{*} \phi(t)\right\rangle_{H} d t=\int_{0}^{\tau}\left\langle u(t), B^{*} \phi(t)\right\rangle_{U} d t
$$

Ponemos $\phi(t):=S(\tau-t)^{*} z_{T}$ para cada $z_{T} \in H$. Es claro que $\dot{\phi}(t)+A^{*} \phi(t)=0$. Entonces definimos la solución del problema de Cauchy (6)-(7) como sigue.

Definition Sean $T>0, y_{0} \in H$ у $u \in L^{2}((0, T) ; U)$. Una solución para el problema de Cauchy $(6)-(7)$ es una función $y \in C^{0}([0, T] ; H)$ tal que

$$
\langle y(\tau), \phi(\tau)\rangle_{H}-\left\langle y_{0}, \phi(0)\right\rangle_{H}=\int_{0}^{\tau}\left\langle u(t), B^{*} S(\tau-t) z_{T}\right\rangle_{U} d t
$$

para cualesquiera $\tau \in[0, T], z_{T} \in H$.

Por la propiedad de regularidad del operador $B$, el lado derecho de la igualdad $(8)$ esta bien definido. 
Theorem 5. Fijamos $T>0$. Para cualesquiera $y_{0} \in H$ y $u \in L^{2}((0, T) ; U)$, el problema de Cauchy (6)-(7) tiene una única solución y. Más aún, existe una constante $C=C(T)>0$, independiente de $y_{0} y$ u, tal que

$$
\|y(\tau)\|_{H} \leq C\left[\left\|y_{0}\right\|_{H}+\|u\|_{L^{2}((0, T) ; U)}\right] \text { para todo } \tau \in[0, T]
$$

\section{IV | APLICACIONES}

En esta sección queremos mostrar como la teoría de semigrupos fuertemente continuos de operadores lineales continuos se aplica a la prueba de existencia y unidad de soluciones para la ecuación de transporte en una dimensión y cierta ecuación de transporte-difusión, las cuales están relacionadas con un problema en teoría de control tratado por Coron y Guerrero en (Guerrero, 2005).

\section{I La ecuación de transporte}

Fijamos $T, L>0$. Consideramos la llamada ecuación de transporte

$$
\left\{\begin{array}{l}
y_{t}+y_{x}=0, t \in(0, T), x \in(0, L), \\
y(t, 0)=u(t) \\
y(0, x)=y_{0}(x), x \in(0, L)
\end{array}\right.
$$

donde $t$ es el tiempo, $u(t) \in \mathbb{R}$ es el control y $y(t, \cdot):(0, L) \rightarrow \mathbb{R}$ es el estado.

Es fácil verificar que la solución para 10 está dada por la función $y(t, x)=\left\{\begin{array}{ll}u(t-x), & 0 \leq x<t \\ y_{0}(x-t), & 0 \leq t<x\end{array}\right.$, siempre que $u$ y $y_{0}$ sean funciones diferenciables.

\section{Problema de Cauchy bien planteado}

Para $T, L>0$, en esta sección damos sentido a la noción de solución débil de la ecuación de transporte

$$
\left\{\begin{array}{l}
y_{t}+y_{x}=0, \quad t \in(0, T), x \in(0, L), \\
y(t, 0)=u(t), \quad t \in(0, T), \\
y(0, x)=y_{0}(x), \quad x \in(0, L)
\end{array}\right.
$$

donde $y_{0} \in L^{2}(0, T), u \in L^{2}(0, T)$ son funciones dadas. Mostraremos que en tal sentido, el problema está bien planteado.

Primero supongamos que existe $y \in C^{1}([0, T] \times[0, L])$ que satisface $[11)$. Sean $\tau \in[0, T], \phi \in C^{1}([0, \tau] \times$ $[0, L])$, entonces

$$
\int_{0}^{\tau} \int_{0}^{L}\left[\phi y_{t}+\phi y_{x}\right] d x d t=0
$$

Si integramos por partes obtenemos

$$
\begin{aligned}
\left.\int_{0}^{L} \phi y\right|_{0} ^{\tau} d x+\left.\int_{0}^{\tau} \phi y\right|_{0} ^{L} d t-\int_{0}^{\tau} \int_{0}^{L}\left[\phi_{t}+\phi_{x}\right] y d x d t & =\int_{0}^{L}\left[\phi(\tau, x) y(\tau, x)-\phi(0, x) y_{0}(x)\right] d x \\
& +\int_{0}^{\tau}[\phi(t, L) y(t, L)-\phi(t, 0) u(t)] d t \\
& -\int_{0}^{\tau} \int_{0}^{L}\left[\phi_{t}+\phi_{x}\right] y d x d t=0 .
\end{aligned}
$$

Lo anterior motiva la definición de una solución débil de [11. 
Definition Para $T, L>0$, consideramos $y_{0} \in L^{2}(0, L)$ y $u \in L^{2}(0, T)$. Una solución débil de 11 es una función $y \in C^{0}([0, T] \times[0, L])$ tal que para cada $\tau \in[0, T]$ y para toda $\phi \in C^{1}([0, \tau] \times[0, L])$ con $\phi(t, L)=0 t \in[0, \tau]$, satisface que

$$
\int_{0}^{\tau} \int_{0}^{L}\left(\phi_{t}+\phi_{x}\right) y d x d t=\int_{0}^{L} \phi(\tau, x) y(\tau, x) d x-\int_{0}^{L} \phi(0, x) y_{0}(x) d x-\int_{0}^{\tau} \phi(t, 0) u(t) d t .
$$

Cabe destacar que la definición anterior motiva la siguiente observación. Si y es una solución débil de 111) de clase $C^{1}$ en $[0, T] \times[0, L]$, entonces se cumplirá que
I. $y_{0} \in C^{1}([0, L])$,
II. $u \in C^{1}([0, T])$,
III. $y(0, x)=y_{0}(x)$ para todo $x \in[0, L]$,
IV. $y(t, 0)=u(t)$ para todo $t \in[0, T]$,
V. $y_{t}(t, x)+y_{x}(t, x)=0$ para todo $(t, x) \in[0, T] \times$ $[0, L]$.

El siguiente resultado muestra que las soluciones de (11) dependen continuamente de los datos.

Theorem 6. Para $T, L>0$, consideramos $y_{0} \in L^{2}(0, L), u \in L^{2}(0, T)$. Entonces $(11)$ tiene una única solución débil; además dicha solución, denotada por y, satisface que

$$
\|y(\tau, \cdot)\|_{L^{2}(0, L)} \leq\left\|y_{0}\right\|_{L^{2}(0, L)}+\|u\|_{L^{2}(0, T)} \text { para todo } \tau \in[0, T] .
$$

Prueba. Para la existencia, es fácil verificar que tal solución de (11) está dada por la función

$$
y(t, x)=\left\{\begin{array}{l}
u(t-x), \quad(t, x) \in[0, T] \times[0, L], x<t \\
y_{0}(x-t), \quad(t, x) \in[0, T] \times[0, L], x \geq t .
\end{array}\right.
$$

Para deducirla podemos utilizar el método de las características, el cual se puede encontrar en (John. 1991). Una vez que tenemos esta solución es fácil probar la desigualdad. EL resultado general se sigue de la densidad de las funciones diferenciables en $L^{2}$.

Forma alternativa de la prueba de la existencia: Otra forma de probar la existencia de la solución es utilizando la teoría de semigrupos de operadores lineales como sigue.

Caso 1: Consideramos $u \in C^{2}([0, T])$ con $u(0)=0, y_{0} \in H^{1}(0, L)$ con $y_{0}(0)=0$. Definimos el operador lineal

$$
\begin{array}{cl}
A: D(A) \subseteq L^{2}(0, L) & \rightarrow L^{2}(0, L) \\
f & \mapsto A f=-f_{x}
\end{array}
$$

donde $D(A):=\left\{f \in H^{1}(0, L) \mid f(0)=0\right\}$.

Se puede mostrar que $D(A)$ es denso en $L^{2}(0, L)$ y que A es un operador cerrado en el sentido de la definición II.

Notamos que para $f \in D(A)$,

$$
\langle A f, f\rangle=-\int_{0}^{L} f f_{x} d x=-\frac{f^{2}(L)}{2} \leq 0,
$$

esto implica que A es disipativo (ver definición $[I T)$.

Para $f \in D(A), g \in H^{1}(0, L)$ notamos que por integración por partes

$$
\langle A f, g\rangle=-\int_{0}^{L} f_{x} g d x=-f(L) g(L)+\int_{0}^{L} f g_{x} d x .
$$


Definimos el conjunto $D\left(A^{*}\right):=\left\{g \in H^{1}(0, L) \mid g(L)=0\right\}$, entonces el operador $A^{*}$ dado por $A^{*} g=$ $g_{x}, g \in D\left(A^{*}\right)$, es el operador lineal adjunto de A. Para $g \in D\left(A^{*}\right)$, tenemos que

$$
\left\langle A^{*} g, g\right\rangle=\int_{0}^{L} g g_{x} d x=-\frac{g^{2}(0)}{2} \leq 0,
$$

lo cual implica que $A^{*}$ es disipativo.

El teorema 4 asegura la existencia de una función $z \in C^{1}\left([0, T] ; L^{2}(0, L)\right) \bigcap C^{0}\left([0, T] ; H^{1}(0, L)\right)$ tal que

$$
\begin{aligned}
\frac{d z}{d t} & =A z-u^{\prime}(t), \text { en } L^{2}(0, L), \\
z(t, 0) & =0, \quad t \in[0, T], \\
z(0, \cdot) & =y_{0} .
\end{aligned}
$$

Definimos la función $y \in C^{1}\left([0, T] ; L^{2}(0, L)\right) \bigcap C^{0}\left([0, T] ; H^{1}(0, L)\right)$ como

$$
y(t, x):=z(t, x)+u(t) \text { para todo }(t, x) \in[0, T] \times[0, L] .
$$

Veamos que (22) satisface (12).

Fijamos $\tau \in[\overline{0, T}]$ y $\phi \in C^{1}([0, \tau] \times[0, L])$. Notamos por integración en espacios de Sobolev (ver (Evans, 2010) $y(15)$

$$
\begin{aligned}
\int_{0}^{\tau} \int_{0}^{L} \phi_{x}(t, x)[z(t, x)+u(t)] d x d t & =-\int_{0}^{\tau} \int_{0}^{L} \phi(t, x) z_{x}(t, x) d x d t+\int_{0}^{\tau} \phi(t, L) z(t, L) d t \\
& +\int_{0}^{\tau} u(t) \int_{0}^{L} \phi_{x}(t, x) d x \\
& =\int_{0}^{\tau} \int_{0}^{L} \phi(t, x) A(z(t)) d x d t+\int_{0}^{\tau} y(t, L) \phi(t, L) d t \\
& -\int_{0}^{\tau} u(t) \phi(t, 0) d t .
\end{aligned}
$$

Además

$$
\begin{aligned}
\int_{0}^{L} \int_{0}^{\tau} \phi_{t}(t, x)[z(t, x)+u(t)] d t d x & =\int_{0}^{L} \int_{0}^{\tau} \phi_{t}(t, x) z(t, x) d t d x+\int_{0}^{L} u(\tau) \phi(\tau, x) d x \\
& -\int_{0}^{\tau} \int_{0}^{L} \phi(t, x) u^{\prime}(t) d x d t .
\end{aligned}
$$

Para cada $\eta \in L^{2}(0, L)$ por $\sqrt{19}$ se cumple

$$
\frac{d}{d t}\langle z(t), \eta\rangle_{L^{2}(0, L)}=\left\langle\frac{d z}{d t}(t), \eta\right\rangle_{L^{2}(0, L)}=\left\langle A(z(t))-u^{\prime}(t), \eta\right\rangle_{L^{2}(0, L)}
$$

Ahora consideramos el conjunto de funciones

$$
\mathcal{V}=\psi(t) \cdot \eta(x), \text { con } \psi \in C^{1}([0, \tau]), \eta \in C^{1}([0, L]) \text { y } \eta(L)=0
$$

Sea $\phi(t, x)=\psi(t) \eta(x) \in \mathcal{V}$. Para la primera integral a la derecha de 24) por 25) e integración por 
partes tenemos

$$
\begin{aligned}
\int_{0}^{\tau} \int_{0}^{L} \psi^{\prime}(t) \eta(x) z(t, x) d x d t & =\int_{0}^{\tau} \psi^{\prime}(t)\langle z(t), \eta\rangle_{L^{2}(0, L)} d t \\
& =\left.\psi(t)\langle z(t), \eta\rangle_{L^{2}(0, L)}\right|_{0} ^{\tau}-\int_{0}^{\tau} \psi(t) \frac{d}{d t}\langle z(t), \eta\rangle_{L^{2}(0, L)} d t \\
& =\int_{0}^{L} \phi(\tau, x) z(\tau, x) d x-\int_{0}^{L} \phi(0, x) z(0, x) d x \\
& -\int_{0}^{\tau} \int_{0}^{L} \phi(t, x)\left[A(z(t))-u^{\prime}(t)\right] d x d t
\end{aligned}
$$

Se puede ver en (Brezis 2007) que $\mathcal{V}$ es denso en el espacio $C^{1}([0, \tau] \times[0, L])$. Entonces por (21), (23), 24) y 26), para cada $\phi \in C^{1}([0, \tau] \times[0, L])$ tenemos

$$
\begin{aligned}
\int_{0}^{\tau} \int_{0}^{L}\left(\phi_{t}+\phi_{x}\right) y d x d t & =\int_{0}^{L} \phi(\tau, x) y(\tau, x) d x-\int_{0}^{L} \phi(0, x) y_{0}(x) d x+\int_{0}^{\tau} \phi(t, L) y(t, L) d t \\
& -\int_{0}^{\tau} \phi(t, 0) u(t) d t .
\end{aligned}
$$

En particular si tomamos $\phi=\left.y\right|_{[0, \tau] \times[0, L]}$ tenemos

$$
\int_{0}^{\tau}|y(t, L)|^{2} d t+\int_{0}^{L}|y(\tau, x)|^{2} d x=\int_{0}^{\tau}|u(t)|^{2} d t+\int_{0}^{L}\left|y_{0}(x)\right|^{2} d x
$$

y de esta última expresión se sigue (13).

Caso 2: Sean $y_{0} \in L^{2}(0, L)$ y $u \in L^{2}(0, T)$ funciones arbitrarias. Existe una sucesión $\left\{y_{0, n}\right\}_{n \in \mathbb{N}} \subseteq D(A)$ tal que $y_{0, n} \rightarrow y_{0}$ en $L^{2}(0, L)$ cuando $n \rightarrow \infty$. También existe una sucesión $\left\{u_{n}\right\}_{n \in \mathbb{N}} \subseteq C^{2}([0, T])$ tal que $u_{n}(0)=0$ y $u_{n} \rightarrow$ u en $L^{2}(0, T)$ cuando $n \rightarrow \infty$. Para cada $n \in \mathbb{N}$ procedemos como en el caso 1 , entonces existe una sucesión $\left\{y_{n}\right\}_{n \in \mathbb{N}}$ de funciones en $C^{1}\left([0, T] ; L^{2}(0, L)\right) \bigcap C^{0}\left([0, T] ; H^{1}(0, L)\right)$ dada por $y_{n}(t, x):=z_{n}(t, x)+u_{n}(t)$, donde $z_{n}$ satisface

$$
\begin{aligned}
\frac{d z_{n}}{d t} & =A z_{n}-u_{n}^{\prime}(t), \quad \text { en } L^{2}(0, L), \\
z_{n}(t, 0) & =0, \quad t \in[0, T], \\
z_{n}(0, \cdot) & =y_{0, n},
\end{aligned}
$$

además

$$
\left\|y_{n}\right\|_{C^{0}\left([0, T] ; L^{2}(0, L)\right)} \leq\left\|u_{n}\right\|_{L^{2}(0, T)}+\left\|y_{0, n}\right\|_{L^{2}(0, L)}, n \geq 1 .
$$

Más aún, para $n, m \in \mathbb{N}$ procedemos como en el caso anterior para obtener

$$
\left\|y_{n}-y_{m}\right\|_{C^{0}\left([0, T] ; L^{2}(0, L)\right)} \leq\left\|u_{n}-u_{m}\right\|_{L^{2}(0, T)}+\left\|y_{0, n}-y_{0, m}\right\|_{L^{2}(0, L)} .
$$

Es decir $\left\{y_{n}\right\}$ es una sucesión de Cauchy en $C^{0}\left([0, T] ; L^{2}(0, L)\right)$, por lo tanto existe y $\in C^{0}\left([0, T] ; L^{2}(0, L)\right)$ tal que $y_{n} \rightarrow$ y en $C^{0}\left([0, T] ; L^{2}(0, L)\right)$ cuando $n \rightarrow \infty$.

En particular

$$
\begin{aligned}
\int_{0}^{\tau} \int_{0}^{L}\left(\phi_{t}+\phi_{x}\right) y_{n} d x d t & \rightarrow \int_{0}^{\tau} \int_{0}^{L}\left(\phi_{t}+\phi_{x}\right) y d x d t \\
\int_{0}^{L} \phi(\tau, x) y_{n}(\tau, x) d x & \rightarrow \int_{0}^{L} \phi(\tau, x) y(\tau, x) d x
\end{aligned}
$$


Dado que

$$
\int_{0}^{\tau} \int_{0}^{L}\left(\phi_{t}+\phi_{x}\right) y_{n} d x d t=\int_{0}^{L} \phi(\tau, x) y_{n}(\tau, x) d x-\int_{0}^{L} \phi(0, x) y_{0, n}(x) d x-\int_{0}^{\tau} \phi(t, 0) u_{n}(t) d t,
$$

para toda $\phi \in C^{1}([0, \tau] \times[0, L])$ con $\phi(t, L)=0$ para $t \in[0, \tau], n \in \mathbb{N}$, se sigue que y es la solución de (11).

Unicidad Supongamos que $y_{1}, y_{2}$ son soluciones de (11). Notamos que y:= $y_{1}-y_{2}$ es la solución del problema de Cauchy homogéneo

$$
\begin{aligned}
y_{t}+y_{x} & =0, \quad(t, x) \in[0, T] \times[0, L], \\
y(t, 0) & =0, \quad t \in(0, T), \\
y(0, x) & =0, \quad x \in(0, x) .
\end{aligned}
$$

También observamos que si $\phi \in C^{1}([0, \tau] \times[0, L])$ con $\phi(t, L)=0, t \in[0, \tau]$, entonces

$$
\int_{0}^{\tau} \int_{0}^{L}\left[\phi_{t}+\phi_{x}\right] y d x d t=\int_{0}^{L} y(\tau, x) \phi(\tau, x) d x .
$$

Definimos una sucesión de funciones $\left\{f_{n}\right\}_{n \in \mathbb{N}}$ en $C^{1}(\mathbb{R})$ tal que

$$
\left\{\begin{array}{l}
f_{n}=0, \quad \text { en }[L,+\infty), \text { para todo } n \in \mathbb{N} \\
\left.f_{n}\right|_{(0, L)} \rightarrow y(\tau, \cdot) \text { en } L^{2}(0, L) \text { cuando } n \rightarrow \infty
\end{array}\right.
$$

Para $n \in \mathbb{N}$ consideramos $\phi_{n} \in C^{1}([0, \tau] \times[0, L])$ dada por

$$
\phi_{n}=f_{n}(\tau+x-t),(t, x) \in[0, \tau] \times[0, L] .
$$

Notamos que $\phi_{n}(\tau, L)=f_{n}(L)=0$ para toda $n \in \mathbb{N}$ y se satisface que

$$
\phi_{n t}+\phi_{n x}=0 \text { en }[0, \tau] \times[0, L] .
$$

Así tomando $\phi:=\phi_{n}$, en (30) junto con (31), entonces

$$
\int_{0}^{L} y(\tau, x) \phi_{n}(\tau, x) d x=\int_{0}^{L} y(\tau, x) f_{n}(x) d x=0,
$$

por lo tanto,

$$
\lim _{n \rightarrow \infty} \int_{0}^{L} y(\tau, x) f_{n}(x) d x=\int_{0}^{L} y^{2}(\tau, x) d x=0
$$

entonces $y(\tau, x)=0$ para todo $x \in[0, L]$.

\section{2| Una ecuación de transporte-difusión}

Fijamos las constantes $L, T>0, \varepsilon>0$ y $M \neq 0$. Consideramos la siguiente ecuación de transportedifusión:

$$
\left\{\begin{array}{rlrl}
y_{t}+M y_{x}-\varepsilon y_{x x} & =0 & \text { si } & (t, x) \in(0, T) \times(0, L) \\
y(t, 0) & =u(t) & \text { si } \quad t \in(0, T) \\
y(t, L) & =0 & \text { si } \quad t \in(0, T)
\end{array}\right.
$$


donde $\varepsilon$ es el parámetro de viscosidad dado. En esta sección vamos a mostrar que (32) tiene una solución cuando imponemos la condición

$$
y(0, x)=y_{0}(x), x \in[0, L],
$$

con $y_{0} \in L^{2}(0, L)$.

Como una motivación para el estudio del sistema (32)-33) en (Fattorini y Russell, 1971) y (Guerrero, 2005) se ha tratado el problema del costo de controlabilidad a cero al tiempo $T$ para dicha ecuación. Cuando nos referimos a que el sistema es controlable a cero, decimos que para cualquier estado inicial $y_{0} \in L^{2}(0, L)$ existe un control $u \in L^{2}(0, T)$ tal que la solución y del sistema 32-33 satisface

$$
y(T, x)=0 \text { para todo } x \in[0, L] .
$$

Notamos que cuando hacemos tender $\varepsilon \rightarrow 0^{+}$en 32 , obtenemos el problema de transporte 110 , , el cual se puede probar que es controlable al tiempo $T>L /|M|$ a partir de $(14)$, dicho resultado se a buscado emular para la ecuación de transporte-difusión cuando el coeficiente de viscosidad tiende a cero, pero hasta el momento lo que se tiene son algunas cotas para el tiempo $T$ las cuales se pueden mejorar.

Retomando el tema, probaremos la existencia y unicidad de la solución para (32)-(33) utilizando la teoría de semigrupos fuertemente continuos, en especifico aplicamos el teorema 5 Para esto introducimos los operadores $A, B$ y verificamos que satisfacen la definición [II] y la desigualdad (3).

Denotamos por $H_{0}^{1}(0, L):=\left\{\beta \in H^{1}(0, L): \beta(0)=\beta(L)=0\right\}$. Es conocido que $H_{0}^{1}(0, L)$ es un espacio de Hilbert sobre el campo real dotado con el producto interior

$$
\langle\alpha, \beta\rangle_{H_{0}^{1}(0, L)}=\int_{0}^{L} \alpha_{x} \beta_{x} d x
$$

Denotamos por $H^{-1}(0, L)$ el dual normado de $H_{0}^{1}(0, L)$. Dado $f \in H^{-1}(0, L)$, por el teorema de representación de Riesz existe $\alpha \in H_{0}^{1}(0, L)$ tal que

$$
f(\beta)=\langle\beta, \alpha\rangle_{H_{0}^{1}(0, L)} \text { para todo } \beta \in H_{0}^{1}(0, L) .
$$

Por otro lado,

$$
f(\varphi)=\int_{0}^{L} \alpha_{x} \varphi_{x} d x=-\alpha_{x x}(\varphi) \text { para todo } \phi \in \mathcal{D}(0, L) .
$$

Como $\overline{\mathcal{D}(0, L)}=H_{0}^{1}(0, L)$ y $\alpha_{x x} \in H^{-1}(0, L)$ se sigue que $f \equiv-\alpha_{x x}$.

Definimos al siguiente mapeo sobreyectivo

$$
\begin{array}{ccc}
J: \quad H^{-1}(0, L) & \rightarrow & H_{0}^{1}(0, L) \\
f & \mapsto & \alpha
\end{array}
$$

De (37) se cumple

$$
\begin{aligned}
J \alpha_{x x} & =-\alpha \text { para todo } \alpha \in H_{0}^{1}(0, L) \\
(J f)_{x x} & =-f \text { para todo } f \in H^{-1}(0, L) \\
\left(J f_{x}\right)_{x} & =-f \text { para todo } f \in L^{2}(0, L)
\end{aligned}
$$


Dotamos al espacio $H^{-1}(0, L)$ con el siguiente producto escalar:

$$
\langle f, g\rangle_{H^{-1}(0, L)}:=\int_{0}^{L}(J f)_{x}(J g)_{x} d x=\langle J f, J g\rangle_{H_{0}^{1}(0, L)}, f, g \in H^{-1}(0, L) .
$$

Por lo tanto, $J$ es un isomorfismo isométrico entre los espacios de Hilbert $H^{-1}(0, L)$ y $H_{0}^{1}(0, L)$.

Consideremos $f \in H^{-1}(0, L), g \in L^{2}(0, L)$. Usando integración por partes y 38 tenemos

$$
\begin{array}{rll}
\langle f, g\rangle_{H^{-1}(0, L)} & = & \langle J f, J g\rangle_{H_{0}^{1}(0, L)} \\
= & \left\langle(J f)_{x},(J g)_{x}\right\rangle_{L^{2}(0, L)} \\
= & -\left\langle J f,(J g)_{x x}\right\rangle_{L^{2}(0, L)} \\
= & \langle J f, g\rangle_{L^{2}(0, L)}
\end{array}
$$

Así

$$
\langle f, g\rangle_{H^{-1}(0, L)}:=\int_{0}^{L}(J f)_{x}(J g)_{x} d x=\int_{0}^{L}(J f) g d x, \text { si } f \in H^{-1}(0, L), g \in L^{2}(0, L) .
$$

Sea $A$ el siguiente operador lineal

$$
\begin{aligned}
& A: \quad D(A) \subset H^{-1}(0, L) \quad \rightarrow \quad H^{-1}(0, L) \\
& f \quad \mapsto \varepsilon f_{x x}-M f_{x},
\end{aligned}
$$

donde $D(A):=H_{0}^{1}(0, L)$. Se puede mostrar que $A$ es un operador densamente definido y cerrado.

Sea $f \in D(A) \cap H^{2}(0, L)$ y $\alpha=J f$, entonces por 40 tenemos

$$
\begin{aligned}
\langle A f, f\rangle_{H^{-1}(0, L)} & =\int_{0}^{L}\left(\varepsilon f_{x x}-M f_{x}\right) \alpha d x \\
& =\varepsilon \int_{0}^{L} \alpha_{x x} f d x+M \int_{0}^{L} \alpha_{x} f d x \\
& =-\varepsilon \int_{0}^{L} f^{2} d x-M \int_{0}^{L} \alpha_{x} \alpha_{x x} d x \\
& =-\varepsilon \int_{0}^{L} f^{2} d x-\frac{M}{2}\left[\alpha_{x}^{2}(L)-\alpha_{x}^{2}(0)\right] .
\end{aligned}
$$

Es decir

$$
\langle A f, f\rangle_{H^{-1}(0, L)}=-\varepsilon \int_{0}^{L} f^{2} d x-\frac{M}{2}\left[\alpha_{x}^{2}(L)-\alpha_{x}^{2}(0)\right] .
$$

Si $\alpha \in D(A) \cap H^{2}(0, L)$, existe $\eta \in[0, L]$ tal que $\alpha_{x}(\eta)=0$ debido a que $\alpha(0)=\alpha(L)=0$, entonces la función $\alpha$ alcanza un máximo(mínimo) en $[0, L]$, de donde se cumple para $s \in[0, L]$

$$
\alpha_{x}^{2}(s)= \begin{cases}2\left|\int_{s}^{\eta} \alpha_{x} \alpha_{x x} d x\right| & \text { si } s \in[0, \eta] \\ 2\left|\int_{\eta}^{s} \alpha_{x} \alpha_{x x} d x\right| & \text { si } s \in(\eta, L] .\end{cases}
$$

Por lo tanto,

$$
\alpha_{x}^{2}(s) \leq 2 \int_{0}^{L}\left|\alpha_{x} \alpha_{x x}\right| d x
$$


Para $a, b \geq 0$ se tiene $2 a b \leq a^{2}+b^{2}$, en particular $2 a b \leq \frac{a^{2}}{\varepsilon^{2}}+\varepsilon^{2} b^{2}$ para todo $\varepsilon>0$. Entonces

$$
\alpha_{x}^{2}(s) \leq \frac{\varepsilon}{|M|+1} \int_{0}^{L} f^{2} d x+\frac{|M|+1}{\varepsilon} \int_{0}^{L} \alpha_{x}^{2} d x, s \in[0, L]
$$

Consideramos el caso cuando $M>0$. Por (44) con $s=0$ tenemos

$$
\begin{aligned}
\frac{M}{2} \alpha_{x}^{2}(0) & \leq \frac{M}{2(M+1)} \varepsilon \int_{0}^{L} f^{2} d x+\frac{M(M+1)}{2 \varepsilon} \int_{0}^{L} \alpha_{x}^{2} d x \\
& \leq \varepsilon \int_{0}^{L} f^{2} d x+\frac{M(M+1)}{\varepsilon} \int_{0}^{L} \alpha_{x}^{2} d x+\frac{M}{2} \alpha_{x}^{2}(L)
\end{aligned}
$$

Por lo tanto,

$$
\langle A f, f\rangle_{H^{-1}(0, L)}=-\varepsilon \int_{0}^{L} f^{2} d x-\frac{M}{2} \alpha_{x}^{2}(L)+\frac{M}{2} \alpha_{x}^{2}(0) \leq \frac{M(M+1)}{\varepsilon} \int_{0}^{L} \alpha_{x}^{2} d x=M \frac{M+1}{\varepsilon}\|f\|_{H^{-1}(0, L)}^{2} .
$$

Cuando $M<0$ usamos (44) con $s=L$ y multiplicar por $-M / 2$.

Así para $M \neq 0$ tenemos

$$
\langle A f, f\rangle_{H^{-1}(0, L)} \leq|M| \frac{|M|+1}{\varepsilon}\|f\|_{H^{-1}(0, L)}^{2}, \text { para todo } f \in D(A) .
$$

Dado el operador identidad Id en $H^{-1}(0, L)$, definimos el operador $A_{k}=A-k \mathrm{Id}$, para algún $k \in \mathbb{R}^{+}$. Si $k \geq|M| \frac{|M|+1}{\varepsilon}$, entonces $\left\langle A_{k} f, f\right\rangle_{H^{-1}(0, L)} \leq 0$, es decir, $A_{k}$ es disipativo.

Ahora calculamos el operador adjunto de $A$. Dadas $f \in D(A) \bigcap H^{2}(0, L)$ y $g \in H^{1}(0, L)$ tal que $-\varepsilon g(0)+M(J g)_{x}(0)=-\varepsilon g(L)+M(J g)_{x}(L)=0$, en particular $-\varepsilon g+M(J g)_{x} \in H_{0}^{1}(0, L)$. Entonces por 40, e integración en espacios de Sobolev.

$$
\begin{array}{rlrl}
\langle A f, g\rangle_{H^{-1}(0, L)} & = & \int_{0}^{L}\left(\varepsilon f_{x x}-M f_{x}\right) J g d x \\
& =-\int_{0}^{L}\left(\varepsilon f_{x}-M f\right)(J g)_{x} d x \\
& = & \int_{0}^{L}\left(\varepsilon f(J g)_{x x}+M f(J g)_{x}\right) d x \\
& = & \int_{0}^{L}\left(-\varepsilon g+M(J g)_{x}\right) f d x \\
& = & \left\langle f, J\left(J^{-1}\left[-\varepsilon g+M(J g)_{x}\right]\right)\right\rangle_{L^{2}(0, L)} \\
& = & \left\langle f, J^{-1}\left[-\varepsilon g+M(J g)_{x}\right]\right\rangle_{H^{-1}(0, L)},
\end{array}
$$

por lo tanto $g \in D\left(A^{*}\right)$ y $A^{*} g=J^{-1}\left[-\varepsilon g+M(J g)_{x}\right]$.

Para $g \in D\left(A^{*}\right)$, existe $h \in H^{-1}(0, L)$ tal que

$$
\langle A f, g\rangle_{H^{-1}(0, L)}=\langle f, h\rangle_{H^{-1}(0, L)},
$$

para toda $f \in \mathcal{D}(0, L)$. 
Por 40,

$$
\begin{aligned}
\int_{0}^{L} f J h d x & =\int_{0}^{L}\left(\varepsilon f_{x x}-M f_{x}\right) J g d x \\
& =-\int_{0}^{L}\left(\varepsilon f_{x}-M f\right)(J g)_{x} d x \\
& =\varepsilon\left\langle(J g)_{x x}, f\right\rangle_{\mathcal{D}^{\prime}(0, L)-\mathcal{D}(0, L)}+M \int_{0}^{L} f(J g)_{x} d x \\
& =\left\langle-\varepsilon g+M(J g)_{x}, f\right\rangle_{\mathcal{D}^{\prime}(0, L)-\mathcal{D}(0, L)}
\end{aligned}
$$

para toda $f \in \mathcal{D}(0, L)$, por lo tanto $-\varepsilon g+M(J g)_{x}=J h \in H_{0}^{1}(0, l)$ y el resultado se sigue de la parte anterior.

Lo anterior nos permite definir formalmente al operador adjunto de $A$ como sigue

$$
\begin{array}{ccc}
A^{*}: \quad D\left(A^{*}\right) \subset H^{-1}(0, L) & \rightarrow & H^{-1}(0, L) \\
g & \mapsto & J^{-1}\left[-\varepsilon g+M(J g)_{x}\right],
\end{array}
$$

donde $D\left(A^{*}\right):=\left\{g \in H^{1}(0, L):-\varepsilon g(0)+M(J g)_{x}(0)=-\varepsilon g(L)+M(J g)_{x}(L)=0\right\}$.

Se puede probar que $A^{*}$ es densamente definido y cerrado.

Además para $f \in D\left(A^{*}\right) \bigcap H^{2}(0, L)$ procedemos como en 42 y obtenemos

$$
\left\langle A^{*} f, f\right\rangle_{H^{-1}(0, L)}=-\varepsilon \int_{0}^{L} f^{2} d x-\frac{M}{2}\left[\alpha_{x}^{2}(L)-\alpha_{x}^{2}(0)\right],
$$

entonces $A^{*}$ satisface la desigualdad 45 .

Para $k \in \mathbb{R}^{+}$el operador $A_{k}^{*}:=A^{*}-k$ Id es disipativo si $k \geq|M| \frac{|M|+1}{2}$ y es el operador adjunto de $A_{k}$. Sea $k \in R^{+}$que cumple todo lo anterior. Por el teorema 3 el operador $A_{k}$ es el generador infinitesimal de un semigrupo de operadores lineales fuertemente continuos denotado por $S_{k}:=\left\{S_{k}(t): t \geq 0\right\}$ sobre $H^{-1}(0, L)$.

Definimos entonces la familia $S=\{S(t): t \in[0, \infty)\}$ de operadores lineales como sigue

$$
\begin{array}{ccc}
S(t): H & \rightarrow & H \\
f & \mapsto & S(t) f:=\mathrm{e}^{k t} S_{k}(t) f
\end{array}
$$

Claramente $S$ es una familia de operadores lineales fuertemente continua con generador infinitesimal $A$ (ver definiciones 5 y el teorema 2), ya que

I) $S(0) f:=S_{k}(0) f=f$ para todo $f \in H$, es decir $\quad[0, \infty)$. $S(0)=$ Id.

III) Dado $f \in H$. tenemos

II) Para $t_{1}, t_{2} \in \mathbb{R}^{+}$y $f \in H$ tenemos que $\quad \lim _{k\left(t_{1}+t_{2}\right)} \frac{S(t) f-f}{t}=\lim _{t \rightarrow 0^{+}} \mathrm{e}^{k t} . \frac{S_{k}(t) f-f}{t}+$

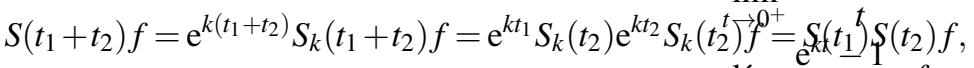
es decir, $S\left(t_{1}+t_{2}\right)=S\left(t_{1}\right) S\left(t_{2}\right)$ para todo $t_{1}, t_{2} \in \quad \lim _{t \rightarrow 0^{+}} \frac{\mathrm{e}^{\lambda}-\underline{1}}{t} \cdot f=(A-k \mathrm{Id}) f+k f=A f$.

Ahora llega el turno de definir al operador $B$. Como motivación para su definición consideramos $f \in H^{2}(0, L)$ con $f(L)=0$ y $g \in D\left(A^{*}\right)$. Entonces usando 38 , 39, e integración por partes en 
espacios de Sobolev tenemos

$$
\begin{array}{rlrl}
\left\langle\varepsilon f_{x x}-M f_{x}, g\right\rangle_{H^{-1}(0, L)} & & \left\langle\varepsilon f_{x x}-M f_{x}, J g\right\rangle_{L^{2}(0, L)} \\
& = & - & \left\langle\varepsilon f_{x}-M f,(J g)_{x}\right\rangle_{L^{2}(0, L)} \\
& = & \varepsilon\left\langle f,(J g)_{x x}\right\rangle_{L^{2}(0, L)}+\left\langle f,-M(J g)_{x}\right\rangle_{L^{2}(0, L)}+\varepsilon f(0)(J g(0))_{x} \\
= & \left\langle f, J A^{*} g\right\rangle_{L^{2}(0, L)}+\varepsilon f(0)(J g(0))_{x} \\
= & \left\langle f, A^{*} g\right\rangle_{H^{-1}(0, L)}+\varepsilon f(0)(J g(0))_{x}
\end{array}
$$

Así por 40) y 46

$$
\left\langle\varepsilon f_{x x}-M f_{x}, g\right\rangle_{H^{-1}(0, L)}=\varepsilon f(0)(J g)_{x}(0)+\left\langle\left(f, A^{*} g\right\rangle_{H^{-1}(0, L)} .\right.
$$

De la definición A.11, si $y \in C^{1}\left([0, T] ; H^{-1}(0, L)\right) \bigcap C^{0}\left([0, T] ; H^{1}(0, L)\right)$ es una solución de $\sqrt[32]{2}$ y si $z \in C^{1}\left([0, T] ; H^{-1}\right) \bigcap C^{0}([0, T] ; D(A))$ es una solución de $z_{t}=A^{*} z$. Ponemos $f \equiv y(0), g \equiv z(0)$ en $[0, L]$. Notamos que $f(L)=y(0, L)=0$ y por 490 se cumple

$$
\left\langle\varepsilon f_{x x}-M f_{x}, g\right\rangle_{H^{-1}(0, L)}:=\varepsilon f(0)(J g)_{x}(0)+\left\langle f, A^{*} g\right\rangle_{H^{-1}(0, L)} .
$$

Sea $D\left(A^{*}\right)^{\prime}$ el espacio dual de $D\left(A^{*}\right)$. Definimos el operador lineal $B$ como sigue:

$$
\begin{aligned}
B: \mathbb{R} & \rightarrow D\left(A^{*}\right)^{\prime} \\
u & \mapsto T_{u},
\end{aligned}
$$

donde

$$
\begin{array}{ccc}
T_{u}: \quad D\left(A^{*}\right) & \rightarrow & \mathbb{R} \\
g & \mapsto & \varepsilon u(J g)_{x}(0)
\end{array}
$$

Por convención $B g:=(B 1)(g), g \in D\left(A^{*}\right)$. Es claro que B es un operador lineal bien definido y continuo. El operador adjunto de $B$ esta dado por

$$
\begin{array}{rlc}
B^{*}: D\left(A^{*}\right) & \rightarrow & \mathbb{R} \\
g & \mapsto \varepsilon(J g)_{x}(0)
\end{array}
$$

Ahora mostramos que se satisface $(3)$.

Sean $T>0, z_{0} \in D\left(A^{*}\right)$ y $z \in C^{1}\left([0, T] ; H^{-1}(0, L)\right) \bigcap C^{0}([0, T] ; D(A))$ dada por

$$
z(t)=S(t)^{*} z_{0}
$$

Sea $\varphi \in C^{1}\left([0, T] ; H_{0}^{1}(0, L)\right) \cap C^{0}\left([0, T] ; H^{3}(0, L)\right)$ dada por

$$
\varphi(t):=J z(t), t \in[0, T] .
$$

Ponemos $\varphi(t, x):=\varphi(t)(x), x \in(0, L)$. Entonces

$$
\varphi(t, \cdot) \in H_{0}^{1}(0, L) \text { у } \varphi_{x x}(t, \cdot)=(J z(t))_{x x}=-z(t, \cdot)
$$

Claramente

$$
\varphi_{t}(t):=J\left(z_{t}(t)\right)=J\left(A^{*} z(t)\right)=-\varepsilon z(t)+M(J z(t))_{x}=\varepsilon \varphi_{x x}(t)+M \varphi_{x}(t) .
$$

Multiplicamos (56) por $-\varphi_{x x}$ y usamos 55 para obtener

$$
\frac{1}{2} \frac{d}{d t} \int_{0}^{L}\left|\varphi_{x}(t, x)\right|^{2} d x=-\varepsilon \int_{0}^{L}\left|\varphi_{x x}(t, x)\right|^{2} d x-\frac{M}{2}\left|\varphi_{x}(t, L)\right|^{2}+\frac{M}{2}\left|\varphi_{x}(t, 0)\right|^{2} .
$$


Usamos 44] con $\alpha:=\varphi(t, \cdot)$, entonces para $s \in[0, L]$ se sigue que

$$
\left|\varphi_{x}(t, s)\right|^{2} \leq \frac{\varepsilon}{|M|+1} \int_{0}^{L}\left|\varphi_{x x}(t, x)\right|^{2} d x+\frac{|M|+1}{\varepsilon} \int_{0}^{L}\left|\varphi_{x}(t, x)\right|^{2} d x .
$$

Supongamos $M>0$. De (57) se sigue que

$$
\frac{d}{d t} \int_{0}^{L}\left|\varphi_{x}(t, x)\right|^{2} d x \leq-2 \varepsilon \int_{0}^{L}\left|\varphi_{x x}(t, x)\right|^{2} d x+M\left|\varphi_{x}(t, 0)\right|^{2}
$$

y utilizando (58) con $s=0$, tenemos

$$
\frac{d}{d t} \int_{0}^{L}\left|\varphi_{x}(t, x)\right|^{2} d x \leq-\varepsilon \int_{0}^{L}\left|\varphi_{x x}(t, x)\right|^{2} d x+M \frac{M+1}{\varepsilon} \int_{0}^{L}\left|\varphi_{x}(t, x)\right|^{2} d x
$$

La desigualdad anterior es válida para $M<0$. En 57) sólo consideramos el término con $\varphi_{x}(t, L)$ y usamos (58).

Por lo tanto, para $M \neq 0$,

$$
\frac{d}{d t} \int_{0}^{L}\left|\varphi_{x}(t, x)\right|^{2} d x \leq-c_{1} \int_{0}^{L}\left|\varphi_{x x}(t, x)\right|^{2} d x+c_{2} \int_{0}^{L}\left|\varphi_{x}(t, x)\right|^{2} d x
$$

con

$$
c_{1}:=\varepsilon, \quad c_{2}:=|M| \frac{|M|+1}{\varepsilon}
$$

Ponemos $\eta(t):=\int_{0}^{L}\left|\varphi_{x}(t, x)\right|^{2} d x$. De 59 tenemos

$$
\eta(\tau)-\mathrm{e}^{c_{1} \tau} \eta(0)=\int_{0}^{\tau} \frac{d}{d t}\left[\mathrm{e}^{-c_{2} t} \eta(t)\right] \leq 0 \quad \tau \in[0, T]
$$

por lo tanto

$$
\int_{0}^{L}\left|\varphi_{x}(t, x)\right|^{2} d x \leq \mathrm{e}^{c_{2} T} \int_{0}^{L}\left|\varphi_{x}(0, x)\right|^{2} d x \text { para todo } t \in[0, T] .
$$

Si integramos $[59]$ en $[0, T]$ y usamos 61 obtenemos

$$
\int_{0}^{T} \int_{0}^{L}\left|\varphi_{x x}(t, x)\right|^{2} d x \leq \frac{\mathrm{e}^{c_{2} T}}{c_{1}} \int_{0}^{L}\left|\varphi_{x}(0, x)\right|^{2} d x
$$

Usando (58) con $s=0$, junto con (61) y 62) tenemos

$$
\int_{0}^{T}\left|\varphi_{x}(t, 0)\right|^{2} d x \leq \frac{C_{T}}{\varepsilon^{2}} \int_{0}^{L}\left|\varphi_{x}(0, x)\right|^{2} d x
$$

donde

$$
C_{T}:=\varepsilon^{2}\left(\frac{\varepsilon}{c_{1}|M|+c_{1}}+T \frac{|M|+1}{\varepsilon}\right) \mathrm{e}^{c_{2} T} .
$$

Por lo tanto se satisface la condición (3) que es equivalente a (63), dado que

$$
\left\|z_{0}\right\|_{H^{-1}(0, L)}=\left\|(J z(0))_{x}\right\|_{L^{2}(0, L)}=\left\|\varphi_{x}(0, x)\right\|_{L^{2}(0, L)} \text { y } B^{*} z(t)=\varepsilon(J z)_{x}(0)=\varepsilon \varphi_{x}(t, 0) .
$$

Así, por el teorema 5 existe una única solución para (32)-(33). 


\section{I CONCLUSIONES}

En este trabajo se presentaron las ideas generales de la teoría de semigrupos de operadores fuertemente continuos, así como su aplicación a las ecuaciones diferenciales. En particular utilizando esta estrategia no clásica se probó la existencia y unicidad de la solución tanto de la ecuación de transporte (10), como de la ecuación de transporte-difusión (32). La estrategia que hemos seguido comenzó construyendo el generador infinitesimal $A$ en cada caso, para así aplicar los resultados de esta teoría, además de eso se destaca que en muchos problemas de teoría de control (ver (Boyer, 2013, Ervedoza y Zuazua, 2010)) que están ligados a las ecuaciones diferenciales se sigue la misma estrategia, ejemplos de esto último se pueden encontrar en (Galo, 2018, R. J. L. Lions, 2000b; Pazy, 1983, Russell, 1978). No sólo se puede aplicar la teoría de semigrupos de operadores fuertemente continuos a las ecuaciones de éste escrito, sino que también a muchas otras, como ser la ecuación de calor, o la ecuación de la onda, o la ecuación de Schrödinger las cuales constituyen ejemplos clásicos en esta área (ver (R. J. L. Lions, 2000b; Pazy, 1983)).

\section{REFERENCIAS}

Bird, R., Stewart, W., y Lightfoot, E. (2007). Fenomenos de transporte. Editorial Limusa S.A. De C.V.

Boyer, F. (2013, diciembre). In the penalised hum approach and its applications to the numerical approximation of null-controls for parabolic problems. ESAIM: Proceedings, 41, pp. 15-58. (44 pages)

Brezis, H. (2007). Analisis funcional, teoría y aplicaciones. Alianza Editorial Sa.

Curtain, R. F. (1978). Infinite dimensional linear systems theory. Springer-Verlag Berlin Heidelberg.

Ervedoza, S., y Zuazua, E. (2010, 01). Sharp observability estimates for heat equations. , 202, 975-1017.

Evans, L. (2010). Partial differential equations. American Mathematical Society.

Fattorini, H. O., y Russell, D. L. (1971). Exact controllability theorems for linear parabolic equations in one space dimension. Archive for Rational Mechanics and Analysis, 43(4), 272-292.

Galo, L. (2018). Tesis: Controlabilidad uniforme a cero de una ecuación de transporte difusión. UNAH 2018.

Guerrero, J. S. (2005). Singular optimal control: A linear 1-d parabolic-hyperbolic example, asymptot. Asymptot, 44(3,4), 237-257.

John, F. (1991). Partial differential equations. Springer New York.

Johnson, C. (2009). Numerical solutions of partial differential equations by the finite element method. Dover Publications.

Lions, J. L. (1988). Contrôlabilité exacte, perturbations et stabilisation de systèmes distribués. tome 1,2, . (Vol. 8). Masson.

Lions, R. J. L. (2000a). Mathematical analysis and numerical methods for science and technology: Volume 2: Functional and variational methods. Springer.

Lions, R. J. L. (2000b). Mathematical analysis and numerical methods for science and technology: Volume 5 evolution problems $i$. Springer.

Michael Reed, B. S. (1978). Methods of modern mathematical physics. analysis of operators (Vol. Volume 4). Academic Press.

Pazy, A. (1983). Semigroups of linear operators and applications to partial differential equations. Springer-Verlag.

Russell, D. L. (1978). Controllability and stabilizability theory for linear partial differential equations: Recent progress and open questions. SIAM Review, 20(4), 639-739. 